\title{
Planning analysis for locally advanced lung cancer: dosimetric and efficiency comparisons between intensity-modulated radiotherapy (IMRT), single-arc/partial-arc volumetric modulated arc therapy (SA/PA-VMAT)
}

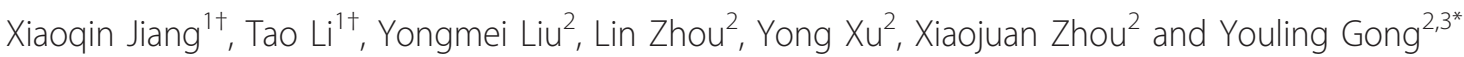

\begin{abstract}
Purpose: To analyze the differences between the intensity-modulated radiotherapy (IMRT), single/partial-arc volumetric modulated arc therapy (SA/PA-VMAT) techniques in treatment planning for locally advanced lung cancer.

Materials and methods: 12 patients were retrospectively studied. In each patient's case, several parameters were analyzed based on the dose-volume histograms (DVH) of the IMRT, SA/PA-VMAT plans respectively. Also, each plan was delivered to a phantom for time comparison.

Results: The SA-VMAT plans showed the superior target dose coverage, although the minimum/mean/maximum doses to the target were similar. For the total and contralateral lungs, the higher $V_{5 / 10}$, lower $V_{20 / 30}$ and mean lung dose (MLD) were observed in the SA/PA-VMAT plans $(p<0.05$, respectively). The PA-VMAT technique improves the dose sparing $\left(V_{20}, V_{30}\right.$ and $\left.M L D\right)$ of the controlateral lung more notably, comparing to those parameters of the IMRT and SA-VMAT plans respectively. The delivered monitor units (MUs) and treatment times were reduced significantly with VMAT plans, especially PA-VMAT plans (for MUs: mean 458.3 vs. 439.2 vs. 435.7 MUs, $p<0.05$ and for treatment time: mean 13.7 vs. 10.6 vs. 6.4 minutes, $p<0.01$ ).

Conclusions: The SA-VMAT technique achieves highly conformal dose distribution to the target. Comparing to the IMRT plans, the higher $V_{5 / 10}$, lower $V_{20 / 30}$ and MLD were observed in the total and contralateral lungs in the VMAT plans, especially in the PA-VMAT plans. The SA/PA-VMAT plans also reduced treatment time with more efficient dose delivering. But the clinical benefit of the VMAT technique for locally advanced lung cancer needs further investigations.
\end{abstract}

Keywords: Lung cancer, Intensity-modulated radiotherapy, Volumetric modulated arc therapy, Target dose distribution, Normal tissue toxicity

\section{Introduction}

VMAT is a new form of IMRT, which allows irradiation with the simultaneously changing gantry position, dose rate and multileaf-collimator (MLC) position [1]. Recently, several studies have been published showing the potential

\footnotetext{
* Correspondence: youlinggong@gmail.com

† Contributed equally

2Department of Thoracic Oncology and Radiation Oncology, Cancer Center, West China Hospital, Sichuan University, Chengdu, Sichuan Province, PR.

China

Full list of author information is available at the end of the article
}

of the VMAT techniques to reduce the treatment time without compromising plan quality compared to IMRT in radiotherapy planning for different cancer types, including lung cancers [2-6], prostate cancer [7] and annal cancer [8]. In practice, the VMAT optimization depends on the choice of various plan parameters, such as the number of arcs, the delivery time or the gantry angle spacing between subsequent control points.

A few studies investigated the clinical and dosimetric advantages of the VMAT technique in treatment for lung cancer presently. Bedford et al. reported the first lung

(C) 2011 Jiang et al; licensee BioMed Central Ltd. This is an Open Access article distributed under the terms of the Creative Commons 
cancer case treated with VMAT [2]. Scorsetti et al. reported the acute toxicity, initial outcome results and planning parameters of 24 patients with the large-volume non-small cell lung cancer (NSCLC) treated with VMAT, while no dosimetric comparison was described [3]. For peripheral lung cancers, McGrath et al. concluded that VMAT allows delivering of the hypofractionated doses much faster than the conventional stereotactic body radiotherapy (SBRT), with the additional advantage for the target dose conformity [4]. Cao et al. compared the plan quality of intensity-modulated arc therapy (IMAT) and helical tomotherapy, and stated that IMAT can provide the plan qualities comparable to that of the helical tomotherapy for most cases [5]. Very recently, Holt's team found that a coplanar VMAT for SBRT for early-stage lung cancer achieved a plan quality and skin dose levels better than those coplanar IMRT plans and reduced treatment time at most by 70\% [6]. To our knowledge, no dosimetric evaluation had been reported between IMRT and VMAT for locally advanced lung cancer yet.

Up to now, the issues existed that whether or not a single arc VMAT could achieve dose distributions comparable to IMRT plan. One study stated that two or more arcs are required in treatment of the complex-shaped target volumes [9], whereas Bertelsen et al. [10] found that a single arc is sufficient to achieve plan quality similar to IMRT. Also, data from Guckenberger et al. indicated the complexity of the target volume determined whether single arc VMAT was equivalent to IMRT [11]. In addition, McGrath's study showed the significant lung dose reduction in VMAT plans appling a partial arc range of $180^{\circ}$ coincide with tumor location [4]. As it could improve lung dose sparing and reducing treatment time, the possible and potential benefits of PA-VMAT in treatment for central and bulky lung cancer are not clear so far.

Here, we reported our planning analysis for the locally advanced lung cancers in these two fields: comparing the dosimetric parameters derived from IMRT and SA/ PA-VMAT plans and evaluating treatment delivery efficiency and treatment times.

\section{Materials and methods}

This study was conducted between October 2010 and March 2011. Totally, 12 patients with pathologically confirmed locally advanced NSCLC were randomly selected for analysis. The patient characteristics were listed in Table 1. All patients were staged according to the modified 1997 AJCC staging system [12]. Permission to conduct the study was granted by the Research Ethics Board of the University Health Network.

\section{Target delineation and dose prescription}

Patients were simulated supine in an individualized thermoplastic mask with their arms raised above the
Table 1 Basic and clinical characteristics of the studied patients $(n=12)$

\begin{tabular}{|c|c|}
\hline \multicolumn{2}{|l|}{ Age (years) } \\
\hline Median & 53 \\
\hline Range & $39-64$ \\
\hline \multicolumn{2}{|l|}{ Sex } \\
\hline Male & 10 \\
\hline Female & 2 \\
\hline \multicolumn{2}{|l|}{ Pathology } \\
\hline Non-small cell lung cancer & 12 \\
\hline \multicolumn{2}{|l|}{ Disease stage } \\
\hline IIla & 3 \\
\hline$\| l b$ & 9 \\
\hline \multicolumn{2}{|l|}{ PTV volume $\left(\mathrm{cm}^{3}\right)$} \\
\hline Median & 221.3 \\
\hline Range & $177.6-412.5$ \\
\hline \multicolumn{2}{|l|}{ Total lung volume $\left(\mathrm{cm}^{3}\right)$} \\
\hline Median & 3525.7 \\
\hline Range & $2676.2-4810.5$ \\
\hline
\end{tabular}

head on a lung board designed to support the elbows. All of the computed tomograph (CT) images (Siemens, Somatom Plus 4) of the patients were transferred to and registered in the treatment planning system (TPS).

The gross tumor volume (GTV) was defined as the macroscopically identifiable tumor including lymph nodes with a diameter more than $1 \mathrm{~cm}$ on $\mathrm{CT}$. The clinical tumor volume (CTV) enclosed the GTV with an $8 \mathrm{~mm}$ margin towards lung tissue and a $5 \mathrm{~mm}$ margin around affected lymph nodes. For the planning target volume (PTV), the 10 or $15 \mathrm{~mm}$ margin was added isotropically to the CTV if the tumor location is superior or inferior lobe. The organs at-risk (OARs) included: lungs (ipsilateral and contralateral), spinal cord, esophagus and heart. The planning organ at-risk volumes (PRVs) were extended as $5 \mathrm{~mm}$ to the esophagus and the spinal cord, respectively; all expansion was not applied to the lungs. The dose-volume constraints for the lungs were set as follows: $\mathrm{V}_{20}<30 \%, \mathrm{~V}_{30}<20 \%$ and mean lung dose $<20$ Gy. The maximum dose of $45 \mathrm{~Gy}$ was allowed to the spinal cord (PRV). In addition, the plan optimization was performed with the aim to keep the esophagus PRV dose of $55 \mathrm{~Gy}\left(\mathrm{~V}_{55}\right)$ to $30 \%$ of the organ volume and the heart PRV dose of $40 \mathrm{~Gy}\left(\mathrm{~V}_{40}\right)$ to $50 \%$ of the organ volume.

All generated plans for each patient consisted of 68 Gy to be delivered to PTV in 34 fractions. The objective of planning was to deliver the prescribed dose to at least $95 \%$ of the PTV with a dose range not exceeding $-10 \%$ and $+15 \%$ of the prescribed dose. All plans were generated for the Elekta Beam Modulator (Elekta Oncology Systems, Crawley, UK). 


\section{Treatment planning and optimizing IMRT}

The IMRT optimization was performed by appling Direct Machine Parameter Optimization (DMPO) algorithm in our treatment planning system (Pinnacle ${ }^{3}$, Philips Radiation Oncology Systems, Fitchburg, USA) as described previously [13]. For each plan, an average of 40 segments were used based on 5 (whose angels were $216^{\circ}, 288^{\circ}, 0^{\circ}, 72^{\circ}, 144^{\circ}$, respectively) or 7 (whose angels were $204^{\circ}, 256^{\circ}, 308^{\circ}, 0^{\circ}, 52^{\circ}, 104^{\circ}, 156^{\circ}$, respectively) coplanar beams with the angles depending on the tumor location (Figure 1a). In the plan generation, the maximum iterations in the plan optimizing were 40 , and the maximum number of all segments in one plan was restricted to 100 . There is no limitation to the MUs per segment. Plans were generated for the Elekta Beam Modulator with 6-MV.

\section{SA-VMAT}

The VMAT planning was done applying the SmartArc planning algorithm in Pinnacle ${ }^{3 \circledR} 9.0$ (research version, Philips Radiation Oncology Systems, Fitchburg, USA). The optimiser (single arc) was constrained to use one single $360^{\circ}$ arc which consisted of 90 control points. The arc was represented by 89 beams each separated by $4^{\circ}$ (Figure $1 \mathrm{~b}$ ), which started and ended at $180^{\circ}$, respectively. The accelerator used automatic dose rate selection which ensures that the maximal possible dose rate was chosen for each individual segment of the arc. The initial step is performed based on SmartArc algorithm to obtain the optimal modulated fluence. In the second step, the segments are optimized based on the small ares of targets with insuffient irradiation dose using the same algorithm. Plans were generated with 6-MV either.

\section{PA-VMAT}

The plans were optimized in the same planning system mentioned above. A $180-200^{\circ}$ partial arc was generated for standardization across the studied cases with the range coinciding with the tumor location while avoiding as much of the contralateral lung as possible, which started between $170^{\circ}-180^{\circ}$ and ended between $0^{\circ}-10^{\circ}$.
The arc was represented by 44-49 beams each separated by $4^{\circ}$ either (Figure 1c).

\section{Evaluation of the DVH-based parameters}

The conformity index (CI) and homogeneous index (HI) for PTV was calculated as we described previously [13]. The CI was defined as cover factor (the percentage of the PTV volume receiving $68 \mathrm{~Gy}$ ) $\times$ spill factor (the volume of the PTV receiving the 68 Gy relative to the total prescription dose-volume). The HI was defined the minimum dose in $5 \%$ of the PTV $\left(D_{5}\right) /$ munimum dose in $95 \%$ of the PTV $\left(D_{95}\right)$.

Other parameters were collected from the DVH of these generated plans and compared to each other respectively, including $\mathrm{V}_{5 / 10 / 20 / 30}$ (the percentage volumes which received 5, 10, 20 and 30 Gy respectively) of lungs, average dose $\left(D_{\text {mean }}\right)$ of lungs/heart/ esophagus, $V_{30}$ and $V_{40}$ (the percentage volumes which received 30 and 40 Gy respectively) of heart, $D_{\max }$ and $\mathrm{D}_{5}$ (the maximum dose and the dose that $5 \%$ volume of spinal cord received) of spinal cord and $V_{55}$ (the percentage volumes which received $55 \mathrm{~Gy}$ ) of esophagus.

All plans were transferred to our treatment system for time comparison.

\section{Statistical analysis}

The collected data were analyzed applying "mean \pm standard deviation (SD)" with SPSS software (version 13.0, Chicago, USA). Based on the Wilcoxon's signed rank test, a value of $p<0.05$ was considered to have statistical significance.

\section{Results}

Totally, 36 plans are generated following the protocol and analyzed. The transverse sections of the representative plans with irradiation dose curves of one patient are shown in Figure 2. Obviously, lower dose irradiated to the larger volume of the total lungs were observed in SA/PA-VMAT plans, respectively.

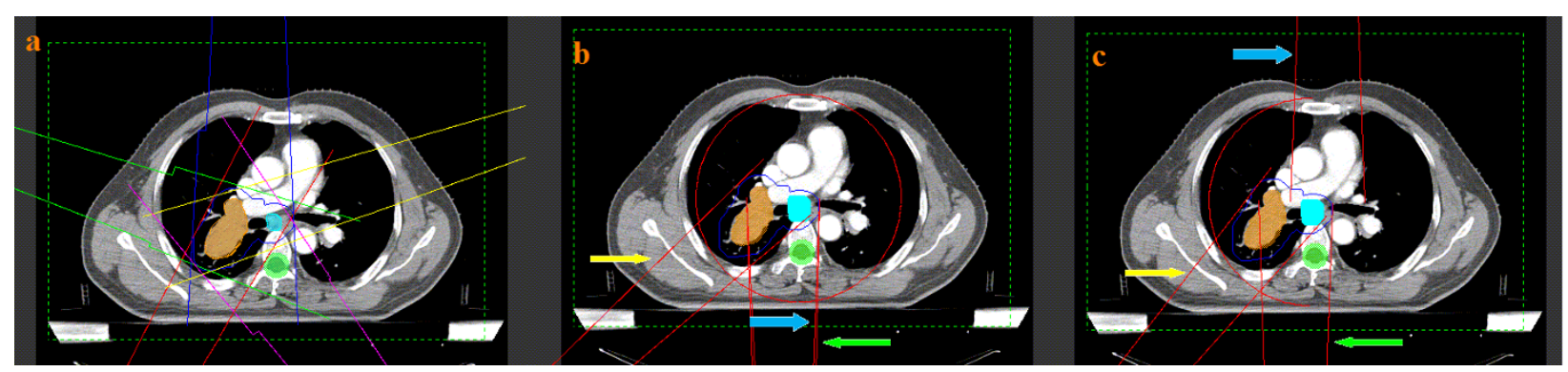

Figure 1 Representative beam arrangements (a: IMRT, b: SA-VMAT and c: PA-VMAT). The green, blue and yellow arrows indicate the start point, end point and one control point in SA/PA-VMAT plans, respectively. 


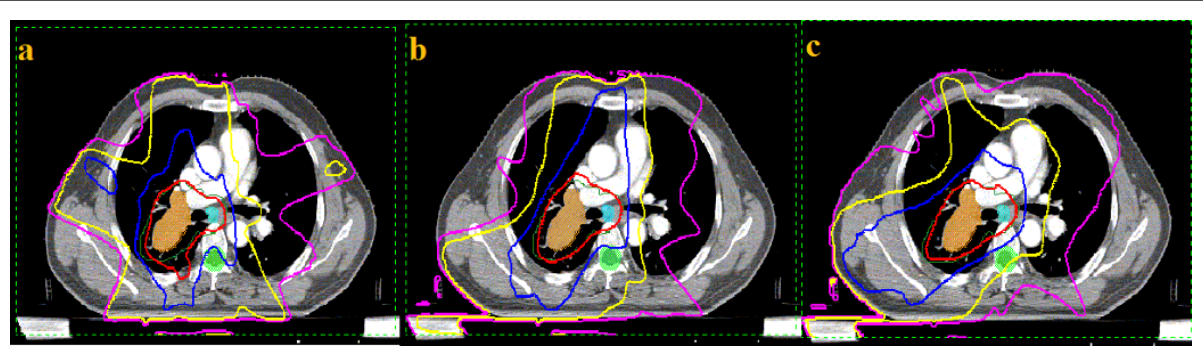

Figure 2 Transverse sections of the representative plans of one patient with the irradiation isodose curves (a: IMRT, b: SA-VMAT and c: PA-VMAT). The pink, yellow, blue and red lines represent the dose curves of 10, 20, 40 and 64.6 (95\% of the prescription dose) Gy,

respectively.

The evaluation of the DVH-based parameters of the PTV is shown in Table 2. The maximum, minimum and average dose of the PTV are similar between IMRT and SA/PA-VMAT plans respectively, with no statistical significance $(p>0.05)$. While, the average CI in SA-VMAT plans $(0.68 \pm 0.06)$ is significantly better than those indexes in plans of IMRT $(0.62 \pm 0.07)$ and PA-VMAT $(0.62 \pm 0.06)$, respectively $(p<0.05)$. Also, the average $\mathrm{HI}$ in SA-VMAT plans $(1.11 \pm 0.04)$ is better than those in IMRT $(1.15 \pm 0.04)$ and PA-VMAT plans $(1.15 \pm$ $0.04)$ respectively, with the statistical significances $(p<$ 0.05).

Table 3 shows the comparisons of the DVH-based parameters of the lungs in the present study. Comparing to the IMRT plans, the SA/PA-VMAT plans show advantages in dose sparing of the total and controlateral lungs, respectively (all $p<0.05$ ). But IMRT plans show the lower $V_{5}$ and $V_{10}$ of the total $(50.6 \pm 10.6 \%$ and 40.2 $\pm 7.2 \%)$ and contralateral lungs $(44.7 \pm 10.7 \%$ and $32.1 \pm$ 7.1\%), comparing to the SA/PA-VMAT plans respectively (all $p<0.05$ ). Although the SA/PA-VMAT plans showing a trend to increaseing the volume received

Table 2 Comparisons of the DVH-based parameters of the PTV in present study $(n=12)$

\begin{tabular}{|c|c|c|c|c|c|c|}
\hline & \multirow{2}{*}{$\begin{array}{l}\text { IMRT } \\
\text { mean } \pm \\
\text { SD }\end{array}$} & \multicolumn{2}{|c|}{ SA-VMAT } & \multicolumn{3}{|c|}{ PA-VMAT } \\
\hline & & $\underset{S D}{\operatorname{mean}} \pm$ & $\begin{array}{c}p \\
\text { value }^{a}\end{array}$ & $\underset{S D}{\operatorname{mean}} \pm$ & $\begin{array}{c}p \\
\text { value }^{b}\end{array}$ & $\begin{array}{c}p \\
\text { value }^{c}\end{array}$ \\
\hline $\begin{array}{l}D_{\min }{ }^{1} \\
(G y)\end{array}$ & $62.4 \pm 1.5$ & $63.2 \pm 1.3$ & 0.179 & $62.5 \pm 1.4$ & 0.908 & 0.217 \\
\hline $\begin{array}{l}D_{\max }{ }^{d} \\
(\mathrm{~Gy})\end{array}$ & $73.9 \pm 1.6$ & $73.4 \pm 1.5$ & 0.492 & $73.8 \pm 1.6$ & 0.919 & 0.555 \\
\hline $\begin{array}{l}D_{\text {mean }}{ }^{d} \\
(G y)\end{array}$ & $68.9 \pm 1.5$ & $68.9 \pm 1.4$ & 0.949 & $68.8 \pm 1.5$ & 0.840 & 0.783 \\
\hline $\mathrm{Cl}^{e}$ & $\begin{array}{l}0.62 \pm \\
0.07\end{array}$ & $\begin{array}{l}0.68 \pm \\
0.06\end{array}$ & 0.019 & $\begin{array}{l}0.62 \pm \\
0.06\end{array}$ & 0.819 & 0.025 \\
\hline $\mathrm{HI}^{f}$ & $\begin{array}{l}1.15 \pm \\
0.04\end{array}$ & $\begin{array}{c}1.11 \pm \\
0.04\end{array}$ & 0.027 & $\begin{array}{c}1.15 \pm \\
0.04\end{array}$ & 0.727 & 0.043 \\
\hline
\end{tabular}

$\overline{a_{\text {: }} \text { compared with the parameters of IMRT; }{ }^{b}: \text { compared with the parameters of }}$ IMRT; ${ }^{c}$ : compared with the parameters of SA-VMAT; ${ }^{d}$ : the minimum, maximum and mean irradiation dose of the PTV, respectively; ${ }^{e}$ : conformity index, calculated with the fomula as we applied previously [13]; ${ }^{\text {: }}$ homogeneous index, calculated with the fomula: "HI = $\mathrm{D}_{5} / \mathrm{D}_{95}{ }^{\prime}$; irradiation, no siginificant difference was observed in the comparisons between the parameters of the ipsilateral lungs in this study (all $p>0.05$ ). Especially, the PAVMAT technique reduced the $\mathrm{V}_{20}(7.5 \pm 2.2), \mathrm{V}_{30}(1.3 \pm$ $0.6)$ and MLD (7.8 \pm 1.0$)$ of the contralateral lungs significantly, comparing to those parameters of the SAVMAT plans ( $p=0.049,0.048$ and 0.038 , respectively).

The comparisons of the parameters of other OARs in present study are also shown in Table 3. The SA/PAVMAT techniques do not show advantages in dose sparing of the other evaluating OARs $\left(D_{\max }\right.$ and $D_{5}$ to the spinal cord; $V_{30}, V_{40}$ and $D_{\text {mean }}$ to the heart; $V_{55}$ and $D_{\text {mean }}$ to the esophagus). These differences between the IMRT and SA/PA-VMAT plans are not statistically significant respectively (all $p>0.05$ ).

Comparing to the IMRT plans $(13.7 \pm 2.6$ minutes $)$, the SA/PA-VMAT plans reduce the treatment time $(10.6 \pm 1.8$ and $6.4 \pm 1.5$ minutes, respectively) significantly as expected (all $p<0.01$ ). Also, we found that the SA/PA-VMAT techniques are more efficient in dose delivery during treatment than that of the IMRT plans $(439.2 \pm 22.5 / 435.7 \pm 25.3$ vs. $458.3 \pm 21.9$ MUs $)$ (shown in Table 4).

\section{Discussion}

As mentioned earlier, the implementation of the VMAT technique gains its value in the treatment for different kinds of the solid tumors, even the tumor targets shaped complex, compared to the conformal radiation therapy (CRT) and IMRT. In present study, we provided in details the dosimetric differences between IMRT and the SA/PA-VMAT plans in the treatment for locally advanced lung cancer.

At present, with the introduction of involved-field radiation therapy (IFRT) and omission of elective nodal irradiation (ENI) in treatment for locally advanced NSCLC, the PTVs of stage III cases, especially IIIB (involving the bilateral mediastinum) are somewhat complex and more like a "dumb bell". Referring to the suggestion from Guckenberger et al. [11], the SAVMAT plans were generated and compard to IMRT 
Table 3 Comparisons of the DVH-based parameters of the $\operatorname{OARs}^{a}$ in present study $(n=12)$

\begin{tabular}{|c|c|c|c|c|c|c|}
\hline & \multirow{2}{*}{$\begin{array}{c}\text { IMRT } \\
\text { mean } \pm \text { SD }\end{array}$} & \multicolumn{2}{|c|}{ SA-VMAT } & \multicolumn{3}{|c|}{ PA-VMAT } \\
\hline & & mean $\pm S D$ & $p$ value $^{b}$ & mean $\pm S D$ & $p$ value $^{c}$ & $p$ value \\
\hline \multicolumn{7}{|l|}{ Total lungs } \\
\hline$V_{5}^{e}(\%)$ & $50.6 \pm 10.6$ & $59.7 \pm 10.8$ & 0.048 & $59.3 \pm 9.1$ & 0.042 & 0.923 \\
\hline$V_{10}{ }^{e}(\%)$ & $40.2 \pm 7.2$ & $46.9 \pm 8.3$ & 0.046 & $46.4 \pm 7.3$ & 0.048 & 0.877 \\
\hline$V_{20}^{e}(\%)$ & $25.7 \pm 5.7$ & $21.1 \pm 4.8$ & 0.044 & $20.3 \pm 4.4$ & 0.016 & 0.675 \\
\hline$V_{30}^{e}(\%)$ & $15.8 \pm 3.4$ & $12.9 \pm 3.2$ & 0.043 & $12.5 \pm 3.2$ & 0.023 & 0.876 \\
\hline $\mathrm{MLD}^{f}(\mathrm{~Gy})$ & $14.4 \pm 2.3$ & $12.5 \pm 2.2$ & 0.047 & $12.3 \pm 2.2$ & 0.028 & 0.785 \\
\hline \multicolumn{7}{|c|}{ Contralateral lungs } \\
\hline$V_{5}(\%)$ & $44.7 \pm 10.7$ & $55.2 \pm 11.9$ & 0.033 & $53.8 \pm 9.4$ & 0.038 & 0.752 \\
\hline$V_{10}(\%)$ & $32.1 \pm 7.1$ & $39.4 \pm 9.8$ & 0.048 & $38.5 \pm 7.3$ & 0.041 & 0.801 \\
\hline$V_{20}(\%)$ & $13.8 \pm 4.4$ & $10.2 \pm 4.1$ & 0.046 & $7.5 \pm 2.2$ & $<0.01$ & 0.048 \\
\hline$V_{30}(\%)$ & $3.3 \pm 1.9$ & $2.0 \pm 1.0$ & 0.048 & $1.3 \pm 0.6$ & $<0.01$ & 0.049 \\
\hline MLD (Gy) & $9.6 \pm 1.2$ & $8.7 \pm 1.0$ & 0.038 & $7.8 \pm 1.0$ & $<0.01$ & 0.038 \\
\hline \multicolumn{7}{|l|}{ Ipsilateral lungs } \\
\hline$V_{5}(\%)$ & $67.6 \pm 10.5$ & $68.9 \pm 11.8$ & 0.778 & $72.4 \pm 13.7$ & 0.346 & 0.052 \\
\hline$V_{10}(\%)$ & $56.8 \pm 9.1$ & $57.3 \pm 9.7$ & 0.898 & $61.1 \pm 11.5$ & 0.321 & 0.167 \\
\hline$V_{20}(\%)$ & $43.9 \pm 5.8$ & $45.2 \pm 6.3$ & 0.604 & $43.1 \pm 5.2$ & 0.725 & 0.383 \\
\hline$V_{30}(\%)$ & $29.8 \pm 1.2$ & $29.9 \pm 1.4$ & 0.853 & $29.5 \pm 1.2$ & 0.547 & 0.460 \\
\hline MLD (Gy) & $18.8 \pm 2.98$ & $20.2 \pm 2.9$ & 0.264 & $18.3 \pm 2.8$ & 0.728 & 0.141 \\
\hline \multicolumn{7}{|l|}{ Spinal cord } \\
\hline$D_{\max }^{f}(G y)$ & $43.0 \pm 2.5$ & $41.6 \pm 2.5$ & 0.194 & $42.5 \pm 2.4$ & 0.603 & 0.404 \\
\hline $\mathrm{D}_{5}{ }^{g}(\mathrm{~Gy})$ & $40.3 \pm 3.8$ & $39.8 \pm 3.9$ & 0.711 & $40.1 \pm 3.9$ & 0.891 & 0.837 \\
\hline \multicolumn{7}{|l|}{ Heart } \\
\hline$V_{30}{ }^{e}(\%)$ & $20.9 \pm 5.7$ & $20.8 \pm 5.8$ & 0.973 & $21.2 \pm 6.3$ & 0.894 & 0.870 \\
\hline$V_{40}^{e}(\%)$ & $14.6 \pm 5.5$ & $13.4 \pm 5.5$ & 0.595 & $15.0 \pm 5.9$ & 0.859 & 0.788 \\
\hline$D_{\text {mean }}^{f}(G y)$ & $14.8 \pm 7.7$ & $14.4 \pm 7.4$ & 0.915 & $15.1 \pm 7.5$ & 0.903 & 0.818 \\
\hline \multicolumn{7}{|l|}{ Esophagus } \\
\hline$V_{55}{ }^{e}(\%)$ & $12.4 \pm 7.3$ & $12.3 \pm 7.5$ & 0.971 & $13.6 \pm 8.3$ & 0.707 & 0.685 \\
\hline$D_{\text {mean }}{ }^{f}(G y)$ & $22.4 \pm 5.6$ & $21.9 \pm 5.2$ & 0.811 & $23.7 \pm 6.3$ & 0.609 & 0.455 \\
\hline
\end{tabular}

${ }^{a}$ : organs at-risk; ${ }^{b}$ : compared with the parameters of IMRT; ${ }^{c}$ : compared with the parameters of IMRT; ${ }^{d}$ : compared with the parameters of SA-VMAT; ${ }^{e}$ : the volume of the lung that received the $5,10,20,30,40$ and 55 Gy irradiation dose, respectively; ${ }^{f}$ : the maximum or mean irradiation dose that the lung, spinal cord, heart and esophagus received, respectively; ${ }^{g}$ : the irradiation dose that the $5 \%$ volume of the spinal cord received.

plans. Under the consideration of reducing more treatment time, we also designed the case-individualized PAVMAT plans for evaluation according to the location of the targets. As expected, our data indicate that in treatment planning for locally advanced lung cancer, a single arc VMAT plan achieve superior dose covarage for PTV (the $\mathrm{CI}$ and $\mathrm{HI}$ are all better, $p<0.05$ ), even a partial arc VMAT could achieve such dose distribution compared to IMRT plans. This was also in a line with the study by Scorsetti et al. [3], the VMAT technique allowed them to achieve the most objectives on target volumes and OARs in radiation therapy for stage III NSCLC in practice. Recently, Yang et al. designed and generated a trajectory-based, noncoplanar subarc for VMAT delivery [14]. They found that after the plan optimization, the trajectory-based VMAT technique showed improved target dose conformality and reduced irradiation dose for OARs, compared to the standard VMAT and IMRT in treating central nervous system tumors. And the potential advantages of such technique

Table 4 Comparisons of other evaluated parameters in present study $(n=12)$

\begin{tabular}{|c|c|c|c|c|c|c|}
\hline & \multirow{2}{*}{$\begin{array}{c}\text { IMRT } \\
\text { mean } \pm \text { SD }\end{array}$} & \multicolumn{2}{|c|}{ SA-VMAT } & \multicolumn{3}{|c|}{ PA-VMAT } \\
\hline & & mean $\pm S D$ & $p$ value $^{a}$ & mean $\pm S D$ & $p$ value $^{b}$ & $p$ value $^{c}$ \\
\hline Delivery time ${ }^{d}$ & $13.7 \pm 2.6$ & $10.6 \pm 1.8$ & $<0.01$ & $6.4 \pm 1.5$ & $<0.01$ & $<0.01$ \\
\hline $\begin{array}{l}\text { Treatment } \\
\text { Efficiency }\end{array}$ & $458.3 \pm 21.9$ & $439.2 \pm 22.5$ & 0.030 & $435.7 \pm 25.3$ & 0.047 & 0.732 \\
\hline
\end{tabular}

${ }^{a}$ : compared with the parameters of IMRT; ${ }^{b}$ : compared with the parameters of IMRT; ${ }^{c}$ compared with the parameters of SA-VMAT; ${ }^{d}$ : presented as minutes; ${ }^{e}:$ presented as monitor units (MUs); 
in radiotherapy for NSCLC need further dosimetric evaluation.

The radiation-induced pneumonitis (RIP) is one of the most common side-effects in CRT for thoracic malignancies. This complication, which has a considerable impact on patient morbidity and sometime leads to death, is strongly correlated with the irradiation dose delivered to the lungs. A number of studies have indicated that the dosimetric parameters from the lung DVH are important in predicting RIP risk [15-18]. Several studies showed that MLD approach seems to provide the most consistent results in terms of increasing RIP rate with increasing MLD, but some stated not $[15,16]$. And many investigations indicated that the $V_{20}$ and $V_{30}$ also were predictive factors of RIP, especially of the severe grade $(\geq 2)[17,18]$. Our study found that the $\mathrm{V}_{20}, \mathrm{~V}_{30}$ and MLD of the total and contralateral lungs were reduced significantly in the SA/PA-VMAT plans than those in IMRT plans respectively $(p<0.05$, as shown in table 3). This founding is correlated with the study of McGrath et al. among lung cancer patients [4], and indicats the possible dosimetric advantages of VMAT in treatment for central and bulky lung cancers. Furthermore, we noticed that the PA-VMAT technique results in significantly better $\mathrm{V}_{20}, \mathrm{~V}_{30}$ and MLD for the contralateral lung when compared to the SA-VMAT technique; while the total lung and ipsilateral lung parameters were not found to be different. Although we realized that SBRT regimens could not easily be compared with conventional CRT regimens, this findings is still partly correlated the results from Holt et al. [6], indicating the potential value of the PA-VMAT technique in treatment for the locally advanced lung cancers.

On the other hand, we noticed that the $V_{5}$ and $V_{10}$ of the total and contralateral lungs in the SA/PA-VMAT plans were higher than those of IMRT plans, respectively $(p<0.05)$. According to the Schallenkamp's report of a 92-patient cohort, the $V_{10 / 13 / 15}$ was also significantly correlated to the RIP either [19]. Wang et al. found that $\mathrm{V}_{5}$ of both lung lobes was the only parameter predicting the RIP ( $\geq$ grade 2 ) in NSCLC patients treated with definitive concurrent chemoradiotherapy [20]. In a study from Netherlands, Palma et al. reported a case of the "Severe" radiological pneumonitis 3 months after SBRT using VMAT and its corresponding treatment plan [21]. Although they concluded that severity and patterns of the RIP were similar, the VMAT techniques still obviously delivered low irradiation dose to a larger volume of lung than IMRT did. From this point of view, the VMAT technique might increase the RIP rate more than the IMRT dose.

The DVH-based parameters of the ipsilateral lung were similar in the IMRT and VMAT plans as displayed in table 3. We could not add the $\mathrm{V}_{5-15}$ and $\mathrm{V}_{20 / 30}$ of the ipsilateral lung as the predictive factors for the RIP following the investigations from Yorke et al. and Ramella et al. [22,23]. As it reduced middle dose volume but increasing low dose volume of the total and contralateral lungs, the conclusion could not be easily obtained that the SA/PA-VMAT techniques had the dosimetric advantages for locally advanced lung cancer from the present study.

In our study, we did not find significant differences in dose sparing of other OARs (spinal cord, heart and esophagus) between the IMRT and SA/PA-VMAT plans in treatment for locally advanced lung cancers.

Beside the dosimetric analysis, similar with other studies mentioned above [3,4,6,9-11], less treatment time was observed in plans delivering using the SA/PAVMAT techniques. This would be another advantage of the VMAT technique: enhancing patient satisfaction and comfort and reducing intrafraction variation. It might potentially help patients tolerate a whole treament procedure, particularly those sick or painful cases. Also in a line with other studies, we observed that the SA/ PA-VMAT plans achieved the comparable dose distribution of the targets with more less MUs, compared to IMRT technique (as shown in table 4).

To our knowdge, the applications of several other techniques that might be helpful to reduce the RIP in radiotherapy for locally advanced lung cancer, including the set-up correction with cone beam CT scan and the active breathing control during simulation and treatment. In present study, we only indicated the dosimetric advantages and disadvantages of the VMAT techniques in treatment for locally advanced lung cancers, compared with the IMRT technique. Even only $18 \%$ grade 1 and $9 \%$ grade 2 acute RIP were observed in 24 patients with large-volume NSCLC after VMAT treatment [3], the potential value of the VMAT techniques for central and bulky lung cancers needs more clinical investigations.

\section{Conclusions}

In this dosimetric and efficiency analysis, the SA-VMAT plans show more optimal target coverage. Compared to the IMRT plans, the VMAT plans reduce the $\mathrm{V}_{20 / 30}$ and MLD but increase the $V_{5 / 10}$ of the total and contralateral lungs. The PA-VMAT technique shows more lung dose sparing of the contralateral lung. With more efficiency, VMAT plans reduce the treatment time significantly. But studies are warranted to evaluate the clinical benefits of the VMAT in treatment for patients with locally advanced NSCLC in future.

\section{Conflict of interest notification}

The authors declare that they have no competing interests. While our radiation physics center is a training 
center for Chinese radiation oncologists and physicists supported by Elekta Oncology Systems (Crawley, UK).

\author{
Author details \\ 'Radiation Physics Center, Cancer Center, West China Hospital, Sichuan \\ University, Chengdu, Sichuan Province, PR.China. ${ }^{2}$ Department of Thoracic \\ Oncology and Radiation Oncology, Cancer Center, West China Hospital, \\ Sichuan University, Chengdu, Sichuan Province, PR.China. ${ }^{3}$ State Key \\ Laboratory of Biotherapy, West China Hospital, Sichuan University, Chengdu, \\ Sichuan Province, PR.China.
}

\section{Authors' contributions}

$X J, T L$ and YG contributed equally in design of the study, collection of data and drafting the manuscript; $Y L, L Z, Y X$ and $X Z$ worked on collection of data and critical revision of the manuscript; YG provided the conception of this study and the final approval of the version to be published. And all authors read and approved the final version of the manuscript.

Received: 10 August 2011 Accepted: 21 October 2011

Published: 21 October 2011

\section{References}

1. Otto K: Volumetric modulated arc therapy: IMRT in a single arc. Med Phys 2008, 35:310-7.

2. Bedford JL, Hansen VN, McNair HA, Aitken AH, Brock JE, Warrington AP, Brada M: Treatment of lung cancer using volumetric modulated arc therapy and image guidance: a case study. Acta Oncol 2008, 47:1438-43.

3. Scorsetti M, Navarria P, Mancosu P, Alongi F, Castiglioni S, Cavina R, Cozzi L, Fogliata A, Pentimalli S, Tozzi A, Santoro A: Large volume unresectable locally advanced non-small cell lung cancer: acute toxicity and initial outcome results with rapid arc. Radiat Oncol 2010, 5:94.

4. McGrath SD, Mathuszak MM, Yan D, Kestin LL, Martinez AA, Grills IS: Volumetric modulated arc therapy for delivery of hypofractionated stereotactic lung radiotherapy: A dosimetric and treatment efficiency analysis. Radiother Oncol 2010, 95:153-7.

5. Cao D, Holmes T, Afghan M, Shepard DM: Comparison of plan quality provided by intensity-modulated arc therapy and helical tomotherapy. Int J Radiat Oncol Biol Phys 2007, 69:240-50.

6. Holt A, van Vroegindeweij C, Mans A, Belderbos JS, Damen EM: Volumetricmodulated arc therapy for stereotactic dosy radiotherapy of lung tumors: a comparison with intensity modulated radiotherapy techniques. Int I Radiat Oncol Biol Phys 2011.

7. Pesce GA, Clivio A, Cozzi L, Nicolini G, Richetti A, Salati E, Valli M, Vanetti E, Fogliata A: Early clinical experience of radiotherapy of prostate cancer with volumetric modulated arc therapy. Radiat Oncol 2010, 5:54.

8. Stieler F, Wolff D, Lohr F, Steil V, Abo-Madyan Y, Lorenz F, Wenz F, Ma S: A fast radiotherapy paradigm for anal cancer with volumetric modulated arc therapy (VMAT). Radiat Oncol 2009, 4:48.

9. Verbakel WF, Cuijpers JP, Hoffmans D, Bieker M, Slotman BJ, Senan S: Volumetric intensity-modulated arc therapy vs. conventional IMRT in head-and-neck cancer: a comparative planning and dosimetric study. Int J Radiat Oncol Biol Phys 2009, 74:252-9.

10. Bertelsen A, Hansen CR, Johansen J, Brink C: Single arc volumetric modulated arc therapy of head and neck cancer. Radiother Oncol 2010, 95:142-8.

11. Guckenberger M, Richter A, Krieger T, Wilbert J, Baier K, Flentje M: Is a single arc sufficient in volumetric-modulated arc therapy (VMAT) for complex-shaped target volumes? Radiother Oncol 2009, 93:259-65.

12. Greene FL, Compton CC, Fritz AG, et al: AJCC cancer staging. Atlas New York: Springer; 2006, 77-88.

13. Gong YL, Wang SC, Zhou L, Liu YM, Xu Y, Lu Y, Bai S, Fu YC, Xu QF, Jiang QF: Dosimetric comparison using different multileaf collimeters in intensity-modulated radiotherapy for upper thoracic esophageal cancer. Radiat Oncol 2010, 5:65.

14. Yang Y, Zhang P, Happersett L, Xiong J, Yang J, Chan M, Beal K, Mageras G, Hunt M: Choreographing couch and collimator in volumetric modulated arc therapy. Int I Radiat Oncol Biol Phys 2011, 80:1238-47.

15. Claude L, Perol D, Ginestet C, Falchero L, Arpin D, Vincent M, Martel I, Hominal S, Cordier JF, Carrie C: A prospective study on radiation pneumonitis following conformal radiation therapy in non-small-cell lung cancer: clinical and dosimetric factors analysis. Radiother Oncol 2004, 71:175-81.

16. Rancati T, Ceresoli GL, Gagliardi G, Schipani S, Cattaneo GM: Factors predicting radiation pneumonitis in lung cancer patients: a retrospective study. Radiother Oncol 2003, 67:275-83.

17. Graham MV, Purdy JA, Emami B, Harms W, Bosch W, Lockett MA, Perez CA: Clinical dose-volume histogram analysis for pneumonitis after 3D treatment for non-small cell lung cancer (NSCLC). Int J Radiat Oncol Biol Phys 1999, 45:323-9.

18. Hernando ML, Marks LB, Bentel GC, Zhou SM, Hollis D, Das SK, Fan M, Munley MT, Shafman TD, Anscher MS, Lind PA: Radiation induced pulmonary toxicity: a dose-volume histogram analysis in 201 patients with lung cancer. Int J Radiat Oncol Biol Phys 2001, 51:650-9.

19. Schallenkamp JM, Miller RC, Brinkmann DH, Foote T, Garces YI: Incidence of radiation pneumonitis after thoracic irradiation: dose-volume correlates. Int J Radiat Oncol Biol Phys 2007, 67:410-6.

20. Wang S, Liao Z, Xie W, Liu HH, Tucker SL, Hu CS, Mohan R, Cox JD, Komaki R: Analysis of clinical and dosimetric factors associated with treatment-related pneumonitis (TRP) in patients with non-small-cell lung cancer (NSCLC) treated with concurrent chemotherapy and threedimensional conformal radiotherapy (3D-CRT). Int I Radiat Oncol Biol Phys 2006, 66:1399-407.

21. Palma DA, Senan $S$, Haasbeek CJA, Verbakel WF, Vincent $A$, Lagerwaard F: Radiological and clinical pneumonitis after stereotactic lung radiotherapy: a matched analysis of three-dimentional conformal and volumetri-modulated arc therapy technique. Int I Radiat Oncol Biol Phys 2011, 80:506-13.

22. Yorke ED, Jackson A, Rosenzweig KE, Braban L, Leibel SA, Ling CC Correlation of dosimetric factors and radiation pneumonitis for nonsmall-cell lung cancer patients in a recently completed dose escalation study. Int I Radiat Oncol Biol Phys 2005, 63:672-82.

23. Ramella S, Trodella L, Claudio Mineo T, Pompeo E, Stimato G, Gaudino D, Valentini V, Cellini F, Ciresa M, Fiore M, Piermattei A, Russo P, Cesario A, D'Angelillo RM: Adding ipsilateral $V_{20}$ and $V_{30}$ to conventional dosimetric constraints predicts radiation pneumonitis in stage IIIA-B NSCLC treated with combined-modality therapy. Int I Radiat Oncol Biol Phys 2010, 76:110-5.

doi:10.1186/1748-717X-6-140

Cite this article as: Jiang et al:. Planning analysis for locally advanced lung cancer: dosimetric and efficiency comparisons between intensitymodulated radiotherapy (IMRT), single-arc/partial-arc volumetric modulated arc therapy (SA/PA-VMAT). Radiation Oncology 2011 6:140.

\section{Submit your next manuscript to BioMed Central and take full advantage of:}

- Convenient online submission

- Thorough peer review

- No space constraints or color figure charges

- Immediate publication on acceptance

- Inclusion in PubMed, CAS, Scopus and Google Scholar

- Research which is freely available for redistribution 